\title{
Dioscin sensitizes cells to TRAIL-induced apoptosis through downregulation of c-FLIP and Bcl-2
}

\author{
YONG-SIK KIM $^{3 *}$, EUN-AE KIM ${ }^{1 *}$, KYU-GUN PARK ${ }^{1 *}$, SUNG-JUN LEE ${ }^{1}$, \\ MI-SUN KIM ${ }^{3}$, HO-YONG SOHN ${ }^{2}$ and TAE-JIN LEE ${ }^{1}$ \\ ${ }^{1}$ Department of Anatomy, College of Medicine, Yeungnam University, 317-1 Daemyung-Dong Nam-Gu, Daegu 705-717; \\ ${ }^{2}$ Department of Food Science and Nutrition, Andong National University, Andong 760-749; ${ }^{3}$ Department of Microbiology \\ and Immunology, School of Medicine, Soonchunhyang University, Cheonan, Chungnam 330-090, Republic of Korea
}

Received May 29, 2012; Accepted July 12, 2012

DOI: $10.3892 /$ or.2012.1962

\begin{abstract}
Tumor necrosis factor-related apoptosis-inducing ligand (TRAIL) has received attention as a potential anticancer drug, because it induces apoptosis in a wide variety of cancer cells but not in most normal human cell types. Here, we showed that co-treatment with subtoxic doses of dioscin and TRAIL-induced apoptosis in Caki human renal cancer cells. Treatment of Caki cells with dioscin downregulated $\mathrm{c}-\mathrm{FLIP}_{\mathrm{L}}$ and $\mathrm{Bcl}-2$ proteins in a dose-dependent manner. Dioscin-induced decrease in $\mathrm{c}-\mathrm{FLIP}_{\mathrm{L}}$ protein levels may be caused by the increased protein instability. We also found that dioscin induced downregulation of Bcl-2 at the transcriptional level. Pretreatment with NAC slightly inhibited the expression levels of $\mathrm{c}-\mathrm{FLIP}_{\mathrm{L}}$ downregulated by the treatment of dioscin, suggesting that dioscin is partially dependent on the generation of ROS for downregulation of c-FLIP ${ }_{L}$. Taken together, the present study demonstrates that dioscin enhances TRAIL-induced apoptosis in human renal cancer cells by downregulation of $\mathrm{c}-\mathrm{FLIP}_{\mathrm{L}}$ and $\mathrm{Bcl}-2$.
\end{abstract}

\section{Introduction}

TRAIL (tumor necrosis factor (TNF)-related apoptosisinducing ligand) belongs to the TNF superfamily, which can induce apoptosis in a wide variety tumor cells but not normal

Correspondence to: Dr Tae-Jin Lee, Department of Anatomy, College of Medicine, Yeungnam University, 317-1, Daemyung-Dong Nam-Gu, Daegu 705-717, Republic of Korea

E-mail: tjlee@med.yu.ac.kr

Dr Ho-Yong Sohn, Department of Food Science and Nutrition, Andong National University, Andong 760-749, Republic of Korea

E-mail: hysohn@andong.ac.kr

*Contributed equally

Key words: dioscin, tumor necrosis factor-related apoptosisinducing ligand, c-FLIP, Bcl-2 cells (1). Because of its ability, TRAIL is showing promise as a cancer therapeutic agent. TRAIL induces apoptosis through interacting with death receptor 4 (DR4; TRAIL-R1) and death receptor 5 (DR5; TRAIL-R2) leading to the formation of the death-inducing signal complex (DISC) with binding of caspase-8, leading to apoptosis (extrinsic or death receptor pathway) $(1,2)$. In addition, TRAIL induces apoptosis via the disruption of the mitochondria membrane permeability, release of cytochrome $\mathrm{c}$ into the cytoplasm and activation of caspase-9 (intrinsic or mitochondria pathway) (3). Despite the beneficial effect of TRAIL to selectively kill tumor cells, many cancer cells appear to show resistance to TRAIL (2). The mechanism of TRAIL resistance is not clearly, but several studies has been reported that TRAIL resistance is intimately associated with overexpression of anti-apoptosis including FADD-like apoptosis regulator (c-FLIP), anti-apoptotic Bcl-2 family proteins (e.g., Bcl-2 and Bcl-xL) and inhibitor of apoptosis proteins (IAPs) (2). However, single treatment with TRAIL may not be sufficient for the treatment of various malignant tumor cells, TRAIL-resistant cancer cells can be sensitized by TRAIL sensitizer such as chemotherapeutic drugs and biochemical inhibitors that suppress the expression of anti-apoptosis-associated proteins including Bcl-2, c-FLIP or XIAP, indicating that combination therapy may be a possibility. Therefore, understanding the molecular mechanisms of TRAIL resistance and ways to sensitize these cells to undergo apoptosis by TRAIL are important issues for effective cancer therapy.

Dioscin, a plant glucoside saponin extracted from the roots of Polygonatum zanlanscianense, has anti-inflammatory, lipid-lowering, anticancer and hepatoprotective effects (4-7). Several mechanisms have been proposed for the anticancer activity of dioscin, including induction of apoptosis and arrest of cell cycle $(8,9)$. Dioscin-induced apoptosis were mediated by activation of caspase- 9 and -3 , together with downregulation of anti-apoptotic Bcl-2 protein $(8,10)$ or by the elevated oxidative stress mediated by downregulation of peroxiredoxins as well as through mitochondria dysfunction $(5,11)$.

The aim of this study is to evaluate dioscin as a sensitizer of TRAIL and to understand the mechanism of the synergy between dioscin and TRAIL against human renal cancer cells. 
Dioscin treatment rendered human renal cancer cells more sensitive to TRAIL. These results suggest that this combined treatment with dioscin and TRAIL may provide a safe and effective therapeutic strategy against malignant cancer that are resistant to various conventional treatments. Furthermore, we provide novel evidence that the prominent sensitizing effect of dioscin on TRAIL-induced apoptosis is due to ROS generation which causes downregulation of c-FLIP.

\section{Materials and methods}

Cells and materials. The Caki cells were obtained from the American Type Culture Collection (ATCC, Rockville, MD, USA). The culture medium used throughout these experiments was Dulbecco's modified Eagle's medium (DMEM), containing $10 \%$ fetal calf serum (FCS), $20 \mathrm{mM}$ HEPES buffer and $100 \mu \mathrm{g} / \mathrm{ml}$ of gentamycin. Anti-Bcl-2, anti-PARP, anti-pro-caspase-3, anti-Mcl-1, and anti-actin antibodies were purchased from Santa Cruz Biotechnology Inc. (Santa Cruz, CA, USA). Anti-c-FLIP antibody was purchased from Alexis Corp. (San Diego, CA, USA). Dioscin was isolated from Polygonatum zanlanscianse PAMP) and were directly added to cell cultures at the indicated concentrations. N-acetyl-Lcysteine (NAC) and pan-caspase inhibitor (Z-VAD-FMK) were purchased from Calbiochem (San Diego, CA, USA).

Purification and identification of dioscin. The root of Dioscorea nipponica Makino was obtained from the Uiseong Medicinal Farm (Uiseong, Korea). Three kilograms of the roots were extracted three times with 5 liters of methanol each time. After filtration, the extract was evaporated in vacuo to give $115 \mathrm{~g}$ of dry sample. The following procedures of purification of dioscin based on silica-gel chromatography were the same as previously reported (12). The purified compound was identified as dioscin by analyses of IR spectroscopy (Perkin-Elmer, Shelton, CT, USA) and 1H- and 13C-NMR spectroscopy (Bruker AMX 300, Rheinsten, Germany).

HPLC analysis of dioscin. The purity of dioscin was confirmed by HPLC analysis as was previously reported (12). Dioscin and its derivatives, such as prosapogenin $\mathrm{A}$ and prosapogenin C were determined by HPLC system comprising an SCL-10A system controller, LC-10AD pump and SPD-10A UV detector (Shimadzu, Japan). The analytical column was a Mightysil RP-C18 GP-250 (Kanto Chemical Co., USA). The mobile phase for HPLC consisted of $75 \%$ acetonitrile (v/v) with a flow rate of $0.7 \mathrm{ml} / \mathrm{min}$. The column temperature was maintained at $30^{\circ} \mathrm{C}$. A $10 \mu \mathrm{l}$ of the sample dissolved in methanol ( $1 \mathrm{mg} / \mathrm{ml}$ ) was injected into the HPLC system, and the UV absorption at $215 \mathrm{~nm}$ was recorded. The retension time of dioscin was $3.25 \mathrm{~min}$ and the purity of dioscin was identified as above $98.5 \%$.

Western blotting. Cellular lysates were prepared by suspending $6 \times 10^{5}$ cells in $100 \mu \mathrm{l}$ of lysis buffer $(137 \mathrm{mM} \mathrm{NaCl}, 15 \mathrm{mM}$ EGTA, $0.1 \mathrm{mM}$ sodium orthovanadate, $15 \mathrm{mM} \mathrm{MgCl}_{2}, 0.1 \%$ Triton X-100, $25 \mathrm{mM}$ Mops, $100 \mu \mathrm{M}$ phenylmethlsulfonyl fluoride, and $20 \mu \mathrm{M}$ leupeptin, adjusted to $\mathrm{pH}$ 7.2). The cells were disrupted by sonication and extracted at $4^{\circ} \mathrm{C}$ for $30 \mathrm{~min}$. Lysates containing proteins were quantified using BCA protein assay kit (Pierce, Rockford, IL, USA). The proteins were electrotransferred to Immobilon-P membranes (Millipore Corp., Bedford, MA, USA). Detection of specific proteins was carried out with an ECL western blotting kit (Millipore) according to the manufacturer's instructions.

Cell count and flow cytometry analysis. Cell counts were performed using a hemocytometer. Approximately $1 \times 10^{6} \mathrm{Caki}$ cells were suspended in $100 \mu \mathrm{l}$ of PBS, and $200 \mu 1$ of $95 \%$ ethanol were added while vortexing. The cells were incubated at $4^{\circ} \mathrm{C}$ for $1 \mathrm{~h}$, washed with PBS, and resuspended in $250 \mu \mathrm{l}$ of $1.12 \%$ sodium citrate buffer ( $\mathrm{pH} 8.4$ ) together with $12.5 \mu \mathrm{g}$ of RNase. Incubation was continued at $37^{\circ} \mathrm{C}$ for $30 \mathrm{~min}$. The cellular DNA was then stained by applying $250 \mu \mathrm{l}$ of propidium iodide $(50 \mu \mathrm{g} / \mathrm{ml})$ for $30 \mathrm{~min}$ at room temperature. The stained cells were analyzed by fluorescent activated cell sorting (FACS) on a FACScanto flow cytometer for relative DNA content based on red fluorescence.

RNA isolation and reverse transcriptase-polymerase chain reaction $(R T-P C R)$. Total cellular RNA was extracted from cells using the Easy-blue Total RNA Extraction kit (iNtRon, Sungnam, Korea). A cDNA was synthesized from $5 \mu \mathrm{g}$ of total RNA using M-MLV reverse transcriptase (Promega, Madison, WI, USA). The cDNAs for c-FLIP, Bcl-2 and actin were amplified by PCR with specific primers. The sequence of the sense primer for c-FLIPL was 5'-CGG ACT ATA GAG TGC TGA TGG-3' and the antisense primers were 5'-GAT TAT CAG GCA GAT TCC TAG-3'. PCR products were analyzed by agarose gel electrophoresis and visualized by ethidium bromide.

Statistical analysis. Three or more separate experiments were performed. Statistical analysis was done by paired Student's t-test or ANOVA. A P-value $<0.05$ was considered to have pronounced difference between experimental and control groups.

\section{Results}

Dioscin treatment induces apoptosis in a dose-dependent manner in Caki cells. To investigate the effect of dioscininduced apoptosis, human renal carcinoma Caki cells were treated with various concentrations of dioscin. Two established criteria were subsequently used to assess apoptosis in this study. Apoptosis was determined in Caki cells using flow cytometry analysis demonstrating hypo-diploid DNA. Fig. 1A shows treatment with dioscin in Caki cells resulted in a markedly increased accumulation of sub-G1 phase in a dose-dependent manner of dioscin. Because cells undergoing apoptosis executed the death program by activating caspases and cleavage of PARP, we analyzed expression levels of procaspase-3, and cleavage of PARP. As demonstrated in Fig. 1B, exposure to dioscin led to a reduction of the 32-kDa precursor, accompanied by a concomitant revealed cleavage of PARP. Next, we analyzed nuclear condensation, which is another hallmark of apoptosis. Combinatory treatment with dioscin plus TRAIL induced nuclear condensation in Caki cells. In contrast, nuclear condensation in Caki cells treated with TRAIL alone or dioscin alone was barely detected. 
A
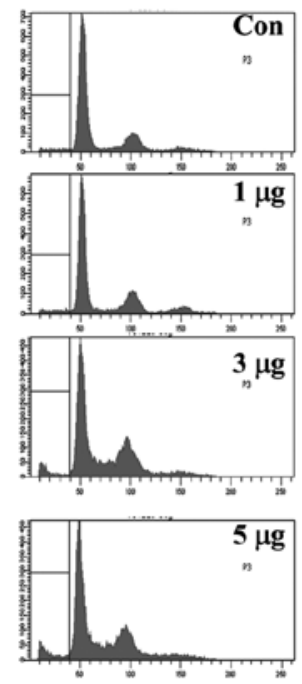

B

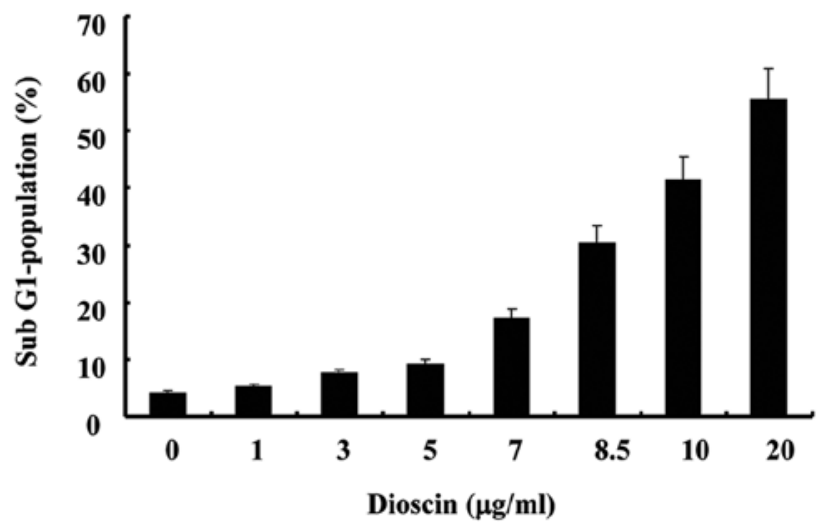

Figure 1. Dioscin treatment induces apoptosis in a dose-dependent manner in Caki cells. (A) Flow cytometric analysis of apoptotic cells (histogram). Caki cells were treated with the indicated concentrations of dioscin for $24 \mathrm{~h}$. Apoptosis was analyzed as a sub-G1 fraction by FACS. (B) Flow cytometric analysis of apoptotic cells (Graph).

$\mathbf{A}$

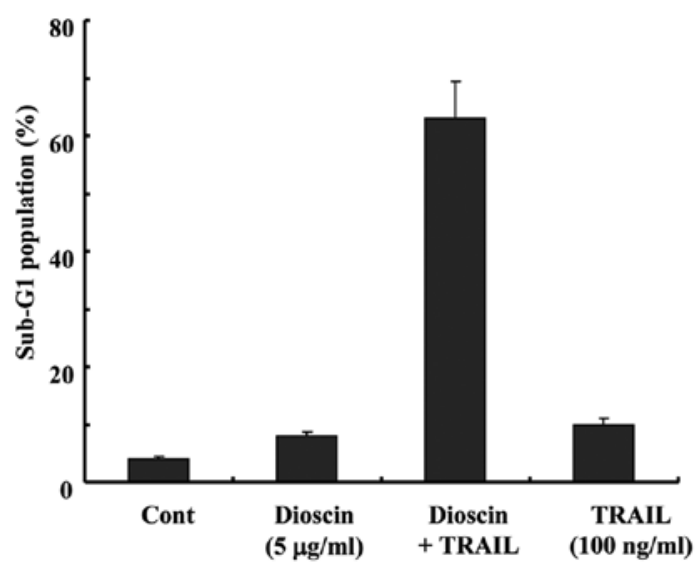

B

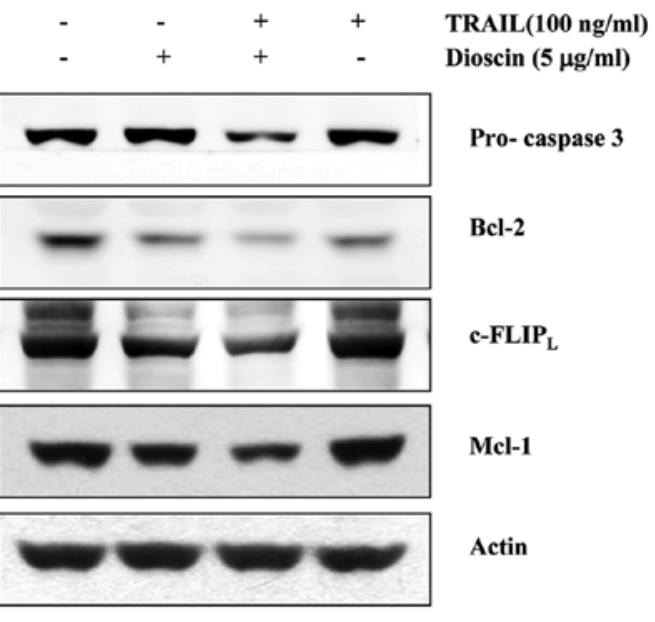

Figure 2. Dioscin sensitizes renal cancer cells to TRAIL-mediated apoptosis. (A) Caki cells were treated for $24 \mathrm{~h}$ with TRAIL (100 ng/ml) in either the absence or the presence of dioscin $(5 \mu \mathrm{g} / \mathrm{ml})$. After $24 \mathrm{~h}$, apoptosis was analyzed as a sub-G1 fraction by FACS. (B) Activation of caspases in dioscin-sensitized TRAIL-induced apoptosis. Cells were treated with the indicated concentrations of dioscin and TRAIL. Equal amounts of cell lysates (40 $\mu$ g) were subjected to electrophoresis and analyzed by western blot analysis for c-FLIP, Bcl-2, Mcl-1 pro-caspase-3, and actin for normalization.

Dioscin sensitizes renal cancer cells to TRAIL-mediated apoptosis. In an attempt to search for novel strategies to overcome TRAIL resistance in cancer cells, we investigated the effect of the combined treatment with dioscin and TRAIL in Caki cells. Co-treatment of Caki cells with dioscin and TRAIL resulted in a markedly increased accumulation of sub-G1 phase cells, compared with Caki cells treated with dioscin or TRAIL alone (Fig. 2A). In addition, combinatory treatment of Caki cells with dioscin and TRAIL strongly stimulated reduction of the protein levels of pro-caspases 3, Bcl-2, Mcl-1, and c-FLIP (Fig. 2B).

Dioscin downregulates Bcl-2, Mcl-1 and c-FLIP protein expressions. To investigate the underlying mechanisms involved in dioscin enhanced TRAIL-induced apoptosis, we analyzed the changes in the expression levels of various apoptosis-regulating proteins. $\mathrm{Bcl}-2, \mathrm{Mcl}-1$ and $\mathrm{c}-\mathrm{FLIP}_{\mathrm{L}}$ protein expressions were decreased by the indicated concentrations of dioscin-treated Caki cells in a dose-dependent manner. To further elucidate the mechanism responsible for the changes in amounts of proteins level, we determined the levels of Bcl-2, Mcl-1 and c-FLIP ${ }_{\mathrm{L}}$ mRNAs by RT-PCR. c-FLIP ${ }_{\mathrm{L}}$ and Mcl-1 mRNA levels remain constant through the dioscin treatment at different doses in Caki cells. We found that dioscin treatment of Caki cells dose-dependently decreased the mRNA levels of Bcl-2 from RT-PCR analysis, suggesting that dioscin modulates Bcl-2 expression at the transcriptional level and $\mathrm{c}-\mathrm{FLIP}_{\mathrm{L}}$ and Mcl-1 at the post-transcriptional level (Fig. 3). 
A

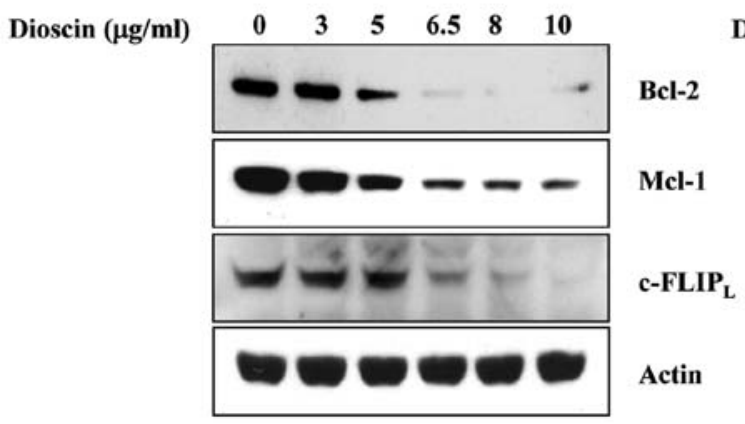

B

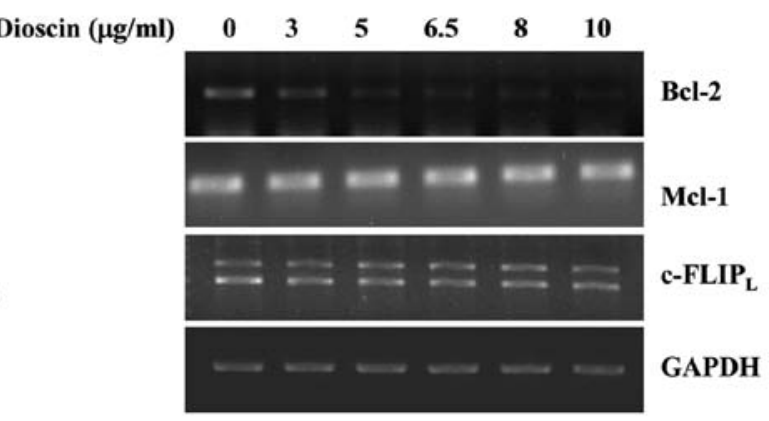

Figure 3. Dioscin modulates Bcl-2 expression at transcriptional level. (A) Caki cells were treated with the indicated doses for $24 \mathrm{~h}$ and harvested in lysis buffer and equal amounts of cell lysates $(40 \mu \mathrm{g})$ were resolved by SDS-PAGE. Western blotting was performed using anti-cFLIP, -Bcl-2, or with anti-actin antibody as a control for the loading of protein level. (B) Caki cells were treated with the indicated concentrations of dioscin for $24 \mathrm{~h}$. Total RNA was isolated and RT-PCR analysis was performed as described in Materials and methods. A representative study is shown; two additional experiments yielded similar results.

$\mathbf{A}$

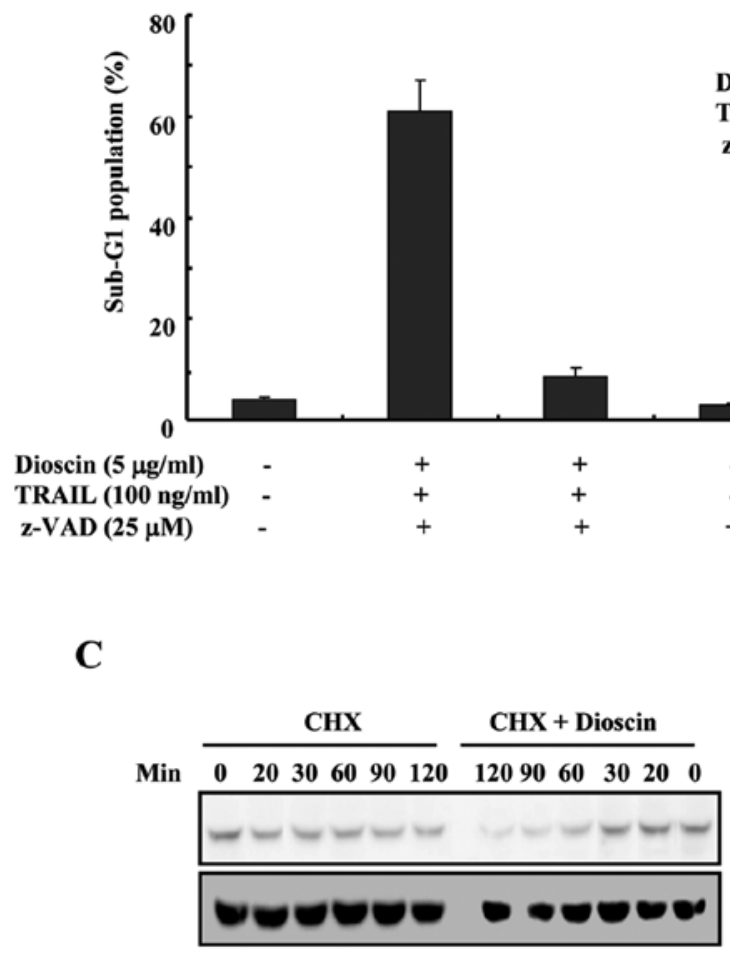

B

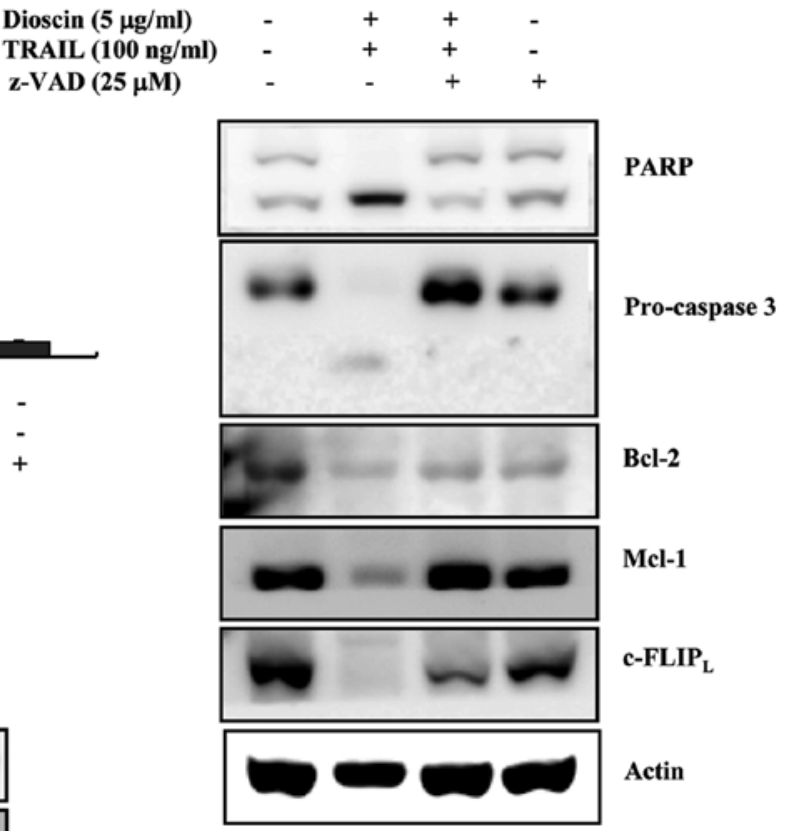

Figure 4. Combinatory treatment with dioscin plus TRAIL-induced apoptosis was mediated via caspase-dependent pathway. (A) Effect of z-VAD-fmk on apoptosis induced by dioscin plus TRAIL. Caki cells were incubated with $50 \mu \mathrm{M} \mathrm{z}$-VAD-fmk or solvent for $1 \mathrm{~h}$ before treatment with dioscin $(5 \mu \mathrm{g} / \mathrm{ml})$ and/or TRAIL ( $100 \mathrm{ng} / \mathrm{ml})$ for $24 \mathrm{~h}$. DNA contents of treated cells were evaluated after propidium iodide staining and apoptosis was measured as a sub-G1 fraction by FACS. (B) Effect of z-VAD-fmk on caspase activation in dioscin plus TRAIL treated cells. Cells were treated with the indicated concentrations of dioscin and TRAIL. Equal amounts of cell lysates $(40 \mu \mathrm{g})$ were subjected to electrophoresis and analyzed by western blot analysis for pro-caspase-3, PARP, c-FLIP, Mcl-1, $\mathrm{Bcl}-2$, and actin to serve as control for the loading of protein level. A representative study is shown; two additional experiments yielded similar results. (C) Caki cells were treated with $5 \mu \mathrm{g} / \mathrm{ml}$ of dioscin in the presence or absence of CHX for the indicated times. Western blotting was performed using anti-c-FLIP antibody and anti-actin antibody served as control for the loading of protein level.

Dioscin plus TRAIL-induced apoptosis was mediated via caspase-dependent pathway. We next examined whether activation of caspase pathway plays a critical role in dioscin plus TRAIL-induced apoptosis. As shown in Fig. 4A, dioscin plus TRAIL-induced apoptosis was completely prevented by pre-treatment with a general and potent inhibitor of caspases, z-VAD-fmk, as determined by FACS analysis. These results suggest that the combined treatment with dioscin and TRAILinduced apoptosis was mediated by caspase-dependent apoptosis pathways. We also found that $\mathrm{z}$-VAD-fmk prevented all these caspase-related events such as cleavage of procaspase-3 and PARP (Fig. 4B). Pretreatment with z-VAD-fmk recovered Mcl-1 protein which were downregulated by combination treatment with dioscon plus TRAIL to basal level, but z-VAD-fmk partly blocked dioscin plus TRAILinduced downregulation of $\mathrm{c}-\mathrm{FLIP}_{\mathrm{L}}$ protein, indicating that the decreased c-FLIP $\mathrm{L}_{\mathrm{L}}$ protein level was partly caused by caspase activation. These results suggested the possibilities that the 
A

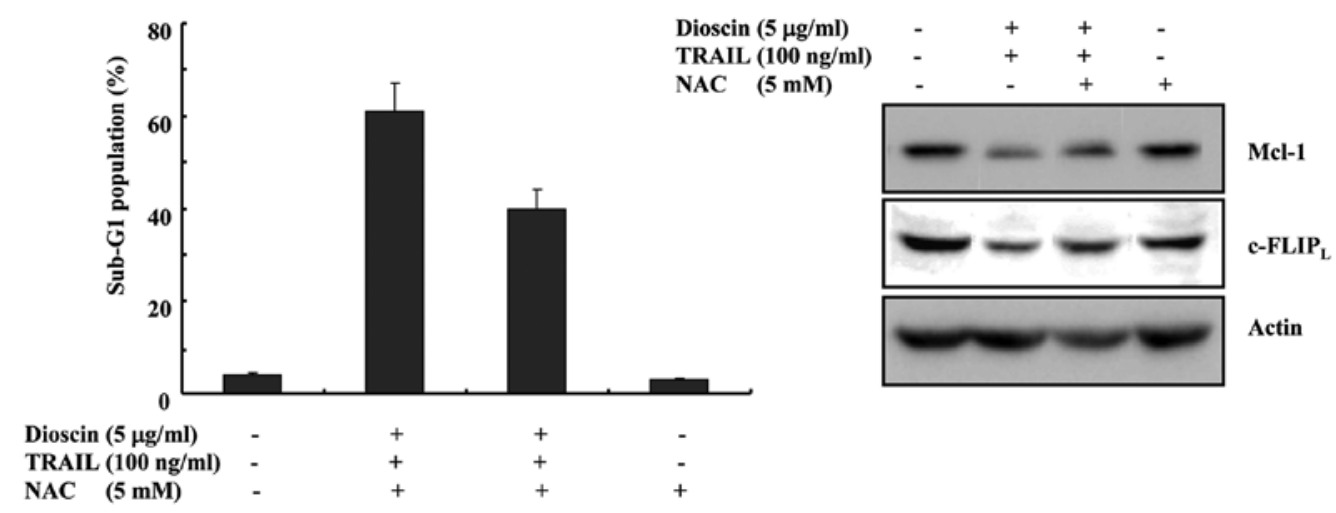

Figure 5. Dioscin-stimulated TRAIL-induced apoptosis appears to be partially dependent on the formation of ROS via downregulation of c-FLIP $\mathrm{L}_{\mathrm{L}}$ (A) Effect of NAC on apoptosis induced by dioscin plus TRAIL. Caki cells were incubated with $5 \mathrm{mM} \mathrm{NAC}$ or solvent for $1 \mathrm{~h}$ before treatment with dioscin $(5 \mu \mathrm{g} / \mathrm{ml})$ and/or TRAIL $(100 \mathrm{ng} / \mathrm{ml})$ for $24 \mathrm{~h}$. DNA contents of treated cells were evaluated after propidium iodide staining and apoptosis was measured as a sub-G1 fraction by FACS. (B) Pretreatment with NAC attenuated dioscin-induced downregulations of c-FLIP ${ }_{\mathrm{L}}$. Western blot analysis was performed as above.

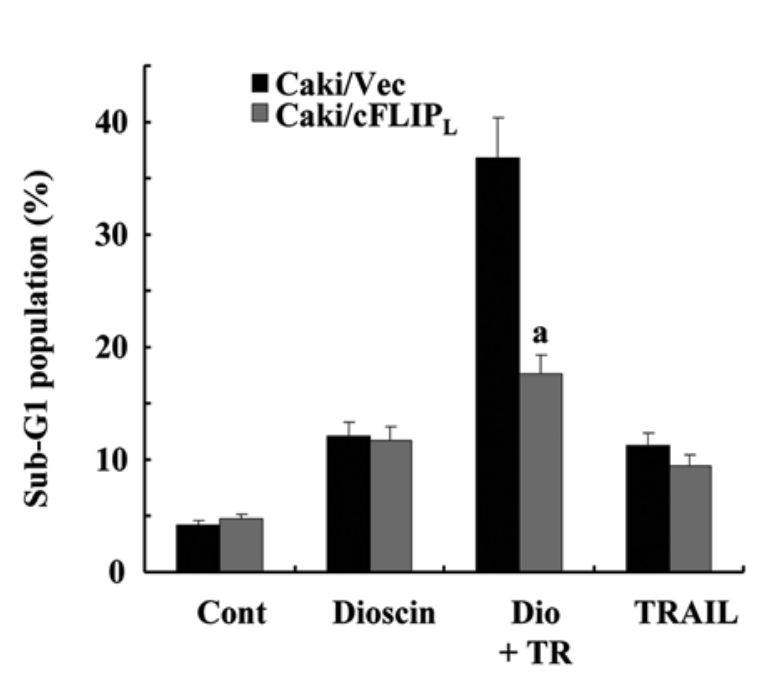

Caki/Vec
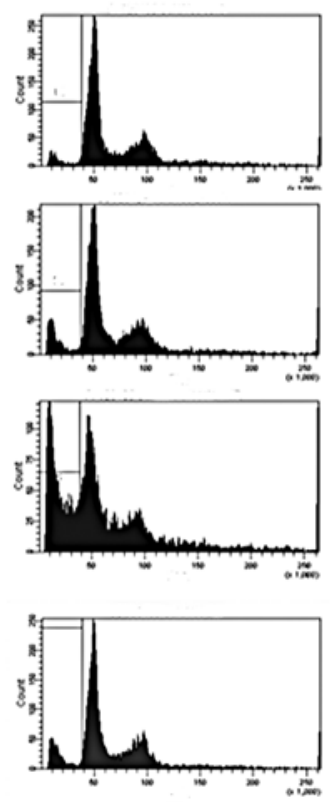

Caki/c-FLIP

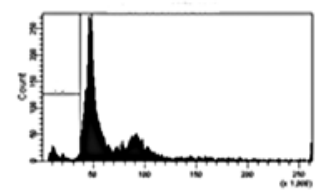

Cont

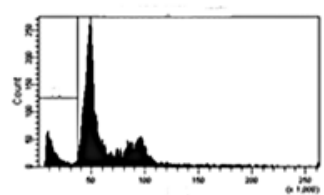

Dioscin

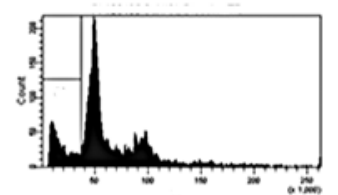

Dioscin

+ TRAIL

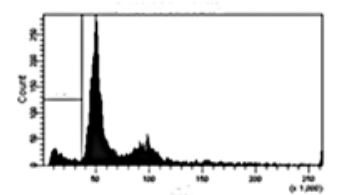

TRAIL

Figure 6. Downregulation of c-FLIP $\mathrm{L}_{\mathrm{L}}$ contributes to dioscin-stimulated TRAIL-induced apoptosis. (A) Caki/Vector and Caki/c-FLIP $\mathrm{L}_{\mathrm{L}}$ cells were treated for $12 \mathrm{~h}$ with dioscin alone $(5 \mu \mathrm{g} / \mathrm{ml})$, TRAIL alone $(100 \mathrm{ng} / \mathrm{ml})$, or combination of dioscin and TRAIL. Apoptosis was analyzed as a sub-G1 fraction by FACS. Data shown are means $\pm \mathrm{SD}(\mathrm{n}=3)$. ${ }^{\mathrm{a}} \mathrm{P}<0.05$ compared to dioscin plus TRAIL-treated pcDNA cells. The FACS data (histogram) are shown in the right panel. (B) Equal amounts of cell lysates $(40 \mu \mathrm{g})$ were subjected to electrophoresis and analyzed by western blot analysis for PARP, procaspase-3, and c-FLIP ${ }_{\mathrm{L}}$.

decreased c-FLIP $\mathrm{L}_{\mathrm{L}}$ protein was partly caused by caspaseindependent pathways (Fig. 4B).

To further clarify the underlying mechanisms of the decreased c-FLIP $\mathrm{L}_{\mathrm{L}}$ protein level in dioscin-treated cells, we performed c-FLIP ${ }_{\mathrm{L}}$ protein stability test. Caki cells were treated with cycloheximide $(\mathrm{CHX})$ and dioscin for different doses. We found that the degradation of $\mathrm{c}-\mathrm{FLIP}_{\mathrm{L}}$ protein was facilitated by dioscin treatment (Fig. 4C), implying that dioscin treatment caused reduction of c-FLIP protein stability.

Dioscin-stimulated TRAIL-induced apoptosis appears to be partially dependent on the formation of reactive oxygen species (ROS) via downregulation of $c-F L I P_{L}$ and Bcl-2. Numerous investigations have documented that ROS may play an important role during apoptosis induction $(13,14)$. It has been reported that dioscin increases ROS production in various cancer cells $(5,10)$. Therefore, we investigated whether ROS generation is directly associated with dioscin plus TRAIL-induced apoptosis. As shown in Fig. 5A, dioscin plus TRAIL-induced apoptosis was completely prevented by pretreatment with NAC, as determined by FACS analysis. As shown in Fig. 5B, pretreatment with NAC decreased the increased expression levels of $\mathrm{c}-\mathrm{FLIP}_{\mathrm{L}}$ and $\mathrm{Bcl}-2$ by dioscin treatment to basal levels, dioscon-induced downregulation of c-FLIPL protein was partly blocked by NAC treatment. 
Downregulations of $c-F L I P_{L}$ contribute to dioscin-stimulated TRAIL-induced apoptosis. We examined whether dowregulation of $\mathrm{c}-\mathrm{FLIP}_{\mathrm{L}}$ by dioscon is critical to stimulate TRAIL-induced apoptosis. Overexpression of $c-F_{L} P_{L}$ in Caki cells significantly attenuated dioscin-facilitated TRAILinduced apoptosis, whereas co-treatment with dioscin plus TRAIL induced significant apoptosis in Caki/vector cells (Fig. 6). This result suggests that c-FLIP ${ }_{\mathrm{L}}$ downregulation also contributes to dioscin-facilitated TRAIL-induced apoptosis.

\section{Discussion}

In this study, we demonstrated for the first time that combination treatment with dioscin plus TRAIL on renal cancer cells synergistically induced apoptosis. Dioscin-mediated dowregulation of $\mathrm{Bcl}-2$ is controlled at the transcriptional level in a dose-dependent manner. In contrast, dioscin-induced downregulation of $\mathrm{c}-\mathrm{FLIP}_{\mathrm{L}}$ is caused by facilitating degradation of

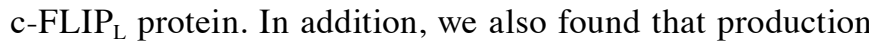
of ROS by dioscin treatment seemed to partially take part in c-FLIP ${ }_{\mathrm{L}}$ downregulation.

Several reagents such as compound $\mathrm{C}$, rosiglitazone, LBH589, and silibinin can induce downregulation of c-FLIP and subsequent sensitization to TRAIL-induced apoptosis in different cancer cells (15-18). It is generally recognized that c-FLIP $\mathrm{L}_{\mathrm{L}}$ protein levels can be regulated by ubiquitin/proteasome mediated degradation $(19,20)$ or by their transcriptional control through the NF- $\kappa \mathrm{B}$ or $\mathrm{c}-\mathrm{Fos}$ pathway $(21,22)$. In this study, dioscin promotes ubiquitin/proteasome-mediated degradation of $\mathrm{c}-\mathrm{FLIP}_{\mathrm{L}}$, leading to downregulation of c-FLIP, but not by transcriptional control. However, further work is needed for the mechanistic study to elucidate dioscin-induced activation of the proteasomal signaling pathway.

It has been suggested that cells can regulate proteasome function in response to increased ROS level both by altering the total number of proteasomes and by altering the subunit components of the ubiquitin-proteasome (23). Dioscin sensitizing HL-60 cells to apoptosis through a ROS-dependent mechanism is supported by direct measurement of ROS generation (5). Recently, several studies have shown that ROS downregulates c-FLIP levels and increases the sensitivity to apoptotic stimuli $(24,25)$. Therefore, we investigated whether downregulations of c-FLIP $\mathrm{L}_{\mathrm{L}}$ was actually mediated by ROS signaling pathway. In the presence of NAC, the decreased levels of $\mathrm{c}-\mathrm{FLIP}_{\mathrm{L}}$ caused by dioscin were partly restored. Taken together, dioscin-stimulated TRAIL-induced apoptosis appears to be dependent on the formation of ROS for downregulations of $\mathrm{c}-\mathrm{FLIP}_{\mathrm{L}}$.

Recently, it has been suggested that cytotoxicity of dioscin was mediated by activating death receptor through upregulation of Fas, FasL (Fas ligand), TNF- $\alpha$, TNF receptor-1, and TNF receptor-associated factor 1 as well as by activating mitochondrial pathways through downregulation of Bcl-2 and in human gastric carcinoma cells (26). However, we found that the expression of Bcl-2 was downregulated by dioscin treatment at transcriptional level in our study, the expression level of TRAIL death receptor (DR5) was not altered by dioscin treatment.

In summary, we suggest that the use of dioscin is a potentially important therapeutic approach for enhancing sensitivity to TRAIL via downregulation of proteins related to the inhibition of the apoptotic processes such as Bcl-2 and c-FLIP.

\section{Acknowledgements}

This research was supported by the Yeungnam University research grants in 2009.

\section{References}

1. Tan ML, Ooi JP, Ismail N, Moad AI and Muhammad TS: Programmed cell death pathways and current antitumor targets. Pharm Res 26: 1547-1560, 2009.

2. Wu GS: TRAIL as a target in anti-cancer therapy. Cancer Lett 285: 1-5, 2009.

3. Deng Y, Lin Y and Wu X: TRAIL-induced apoptosis requires Bax-dependent mitochondrial release of Smac/DIABLO. Genes Dev 16: 33-45, 2002.

4. Sautour M, Mitaine-Offer AC, Miyamoto T, Dongmo A and Lacaille-Dubois MA: A new steroidal saponin from Dioscorea cayenensis. Chem Pharm Bull 52: 1353-1355, 2004.

5. Wang Y, Che CM, Chiu JF and He QY: Dioscin (saponin)-induced generation of reactive oxygen species through mitochondria dysfunction: a proteomic-based study. J Proteome Res 6: 4703-4710, 2007.

6. Kaskiw MJ, Tassotto ML, Mok M, Tokar SL, Pycko R, Th'ng J and Jiang ZH: Structural analogues of diosgenyl saponins: synthesis and anticancer activity. Bioorg Med Chem 17: 7670-7679, 2009.

7. Lu B, Yin L, Xu L and Peng J: Application of proteomic and bioinformatic techniques for studying the hepatoprotective effect of dioscin against $\mathrm{CCl}_{4}$-induced liver damage in mice. Planta Med 77: 407-415, 2011.

8. Cai J, Liu M, Wang Z and Ju Y: Apoptosis induced by dioscin in HeLa cells. Biol Pharm Bull 25: 193-196, 2002.

9. Gao LL, Li FR, Jiao P, et al: Paris chinensis dioscin induces $\mathrm{G} 2 / \mathrm{M}$ cell cycle arrest and apoptosis in human gastric cancer SGC-7901 cells. World J Gastroenterol 17: 4389-4395, 2011.

10. Wang Z, Zhou J, Ju Y, Zhang H, Liu M and Li X: Effects of two saponins extracted from the polygonatum Zanlanscianense pamp on the human leukemia (HL-60) cells. Biol Pharm Bull 24: 159-162, 2001

11. Zhiyu W, Yue C, Neng W, et al: Dioscin induces cancer cell apoptosis through elevated oxidative stress mediated by downregulation of peroxiredoxins. Cancer Biol Ther 13: 138-147, 2012.

12. Kwon CS, Sohn HY, Kim SH, et al: Anti-obesity effect of Dioscorea nipponica Makino with lipase-inhibitory activity in rodents. Biosci Biotechnol Biochem 67: 1451-1456, 2003.

13. Sheng-Tanner X, Bump EA and Hedley DW: An oxidative stress-mediated death pathway in irradiated human leukemia cells mapped using multilaser flow cytometry. Radiat Res 150: 636-647, 1998.

14. Choi YK, Seo HS, Choi HS, Choi HS, Kim SR, Shin YC and Ko SG: Induction of Fas-mediated extrinsic apoptosis, p21WAF1related $\mathrm{G} 2 / \mathrm{M}$ cell cycle arrest and ROS generation by costunolide in estrogen receptor-negative breast cancer cells, MDA-MB-231. Mol Cell Biochem 363: 119-128, 2012.

15. Son YG, Kim EH, Kim JY, et al: Silibinin sensitizes human glioma cells to TRAIL-mediated apoptosis via DR5 up-regulation and down-regulation of c-FLIP and survivin. Cancer Res 67: 8274-8284, 2007.

16. Kim YH, Jung EM, Lee TJ, et al: Rosiglitazone promotes tumor necrosis factor-related apoptosis-inducing ligand-induced apoptosis by reactive oxygen species-mediated up-regulation of death receptor 5 and down-regulation of c-FLIP. Free Radic Biol Med 44: 1055-1068, 2008.

17. Kauh J, Fan S, Xia M, Yue P, Yang L, Khuri FR and Sun SY: c-FLIP degradation mediates sensitization of pancreatic cancer cells to TRAIL-induced apoptosis by the histone deacetylase inhibitor LBH589. PLoS One 5: e10376, 2010.

18. Jang JH, Lee TJ, Yang ES, et al: Compound C sensitizes Caki renal cancer cells to TRAIL-induced apoptosis through reactive oxygen species-mediated down-regulation of c-FLIPL and Mcl-1. Exp Cell Res 316: 2194-2203, 2010.

19. Poukkula M, Kaunisto A, Hietakangas V, et al: Rapid turnover of c-FLIPshort is determined by its unique C-terminal tail. J Biol Chem 280: 27345-27355, 2005. 
20. Kaunisto A, Kochin V, Asaoka T, Mikhailov A, Poukkula M, Meinander A and Eriksson JE: PKC-mediated phosphorylation regulates c-FLIP ubiquitylation and stability. Cell Death Differ 16: 1215-1226, 2009.

21. Li W,Zhang X and Olumi AF: MG-132 sensitizes TRAIL-resistant prostate cancer cells by activating c-Fos/c-Jun heterodimers and repressing c-FLIP(L). Cancer Res 67: 2247-2255, 2007.

22. Benayoun B, Baghdiguian S, Lajmanovich A, et al: NF-kappaBdependent expression of the antiapoptotic factor c-FLIP is regulated by calpain 3 , the protein involved in limb-girdle muscular dystrophy type 2A. FASEB J 22: 1521-1529, 2008.

23. Glickman $\mathrm{MH}$ and Raveh D: Proteasome plasticity. FEBS Lett 579: 3214-3223, 2005
24. Nitobe J, Yamaguchi S, Okuyama M, et al: Reactive oxygen species regulate FLICE inhibitory protein (FLIP) and susceptibility to Fas-mediated apoptosis in cardiac myocytes. Cardiovasc Res 57: 119-128, 2003

25. Kanayama A and Miyamoto Y: Apoptosis triggered by phagocytosis-related oxidative stress through FLIPS down-regulation and JNK activation. J Leukoc Biol 82: 1344-1352, 2007.

26. Hu M, Xu L, Yin L, et al: Cytotoxicity of dioscin in human gastric carcinoma cells through death receptor and mitochondrial pathways. J Appl Toxicol: Feb 14, 2012 (Epub ahead of print). 